\title{
EDITORIAL OPEN \\ Material degradation foreseen in the very long term: the case of glasses and ferrous metals
}

\begin{abstract}
npj Materials Degradation (2017)1:10 ;
doi:10.1038/s41529-017-0008-z
\end{abstract}

Any prediction, even the most rational, even the most scientific, can only be based on an extrapolation from what we have learned and understood from past experience.

Jean-Claude Ameisen

The design of materials which must be in use over extended periods of time, even perhaps greatly exceeding that of human life itself, is one of the challenges faced by engineering and science in general. Such in the case of infrastructure or industrial buildings of steel or reinforced concrete (bridges, nuclear power plants, etc.), whose lifetimes we wish to extend as much as possible, or for the final disposal of nuclear waste in safe environments-a concept which involves interposing a series of different types of barriers between the radionuclides and the biosphere. Engineers are required to predict the behaviour of materials subject to mechanical, chemical, thermal, or other stresses for durations far beyond the range of classical experiments. Among the different issues, one major aspect is the alteration of these materials, which will progressively impact their functional properties (mechanical performance, containment, etc.). It is therefore crucial to be able to satisfactorily model such material degradation, with variable environmental conditions, over extremely long periods of time. Fig. 1

The essential need for long-term prediction represents a significant challenge, and meeting it means drawing on all aspects of today's available experimental science, predictive modelling and engineering. It must also be borne in mind that validating predictions and feedback, can unfortunately only be carried out under laboratory conditions for the practical durations concerned. As such, investigations looking into past situations have become an important part of the overall strategy for predictive approaches implemented, in order to perhaps find some comparisons or even validations on analogous systems. Past situations concern the study of materials produced by humans in ancient civilisations (metals and glasses in our examples) and also of geological materials known as "natural analogues"; in order to work with potentially equivalent materials whose ages are far greater than those possible for anthropogenic materials.

In essence an analogue for anthropogenic materials is not identical in all respects to those for which the behaviour needs to be predicted. This may be because the state of today's materials is known for the initial period (i.e., our time), while the potentially analogous system is only perceptible and measurable now, after a no doubt lengthy alteration period. This means that the analogue approach must compare two systems after having studied them separately, taking into account the fact that these systems are being viewed at very different moments in their history. Only by a number of reviews back and forth between the pieces of information found for these different moments in the life of a system, can a predictive approach be established and consolidated. The theory of such an approach was suggested by Miller et al. ${ }^{1,2}$ as early as the 1990 s. It has been adopted and developed in practice by numerous laboratories, motivated among other things by the special needs of the nuclear industry. ${ }^{3-5}$

Research of analogue materials must focus both on the nature and properties of the material itself (chemical composition, structure at various scales, degree of crystallinity, porosity and so on) and on the conditions of degradation (water composition, temperature, $\mathrm{pH}$; Eh, microbial activity, flow rate and so on). These influencing environmental conditions must be known in details. ${ }^{6}$ In the best cases, most of the influencing parameters remain constant over time; thus limiting the uncertainties on all kind of results issuing from this material.

For both metals and glasses, studies carried out since the 1990s have led to major advances in our understanding of their long term alteration mechanisms. For archaeological objects, the results obtained have also been able to be used in the field of national heritage preservation. For archaeological groups or historical monument managers, the treatments carried out on archaeological objects as they emerge from a dig (for example dechlorination treatment for items in iron) or during their storage in museum reserves, or on monument structures in order to protect them from possible forms of alteration, requires the fullest possible knowledge of altered systems and their potential evolution. Thus work carried out on analogues has been directly applied in these fields in order to optimise these types of treatments. ${ }^{7,8}$ The full interest of such interdisciplinary studies on analogues can be seen through their wide impact into areas which could seem far removed from those strictly concerning the prediction of material behaviour.

\section{THE OVERALL PREDICTION PROCESS}

Predictions for periods lasting centuries or even millennia cannot simply be based on classical semi-empirical approaches (for example, extrapolation based on data produced from systems reconstituted in laboratories). The coupling of generally non-linear phenomena governing material degradation prevents the use of simple extrapolations, and means predictive modelling must be employed. Questions then arise concerning the validation of mechanisms chosen to be taken into account for predictive models.

Archaeological analogues are limited in time by when the materials of interest were first used by humans (typically about 5000 years ago for glasses, about 4000 years ago for copper and about 3000 years ago for iron and low alloy steels). Nevertheless they offer the advantage of allowing the study of materials that are otherwise not found in a natural state to be considered, for example iron alloys. The natural analogues for vitrified radioactive waste are mainly volcanic basaltic or rhyolitic type glasses ${ }^{9}$

Let us examine the case of nuclear glasses, for which material degradation and radionuclides releases predictions for periods lasting several hundreds of millennia are necessary to demonstrate the safety of a deep geological repository. ${ }^{10,11}$ The analogy

Received: 6 January 2017 Revised: 6 February 2017 Accepted: 26 February 2017

Published online: 18 September 2017 


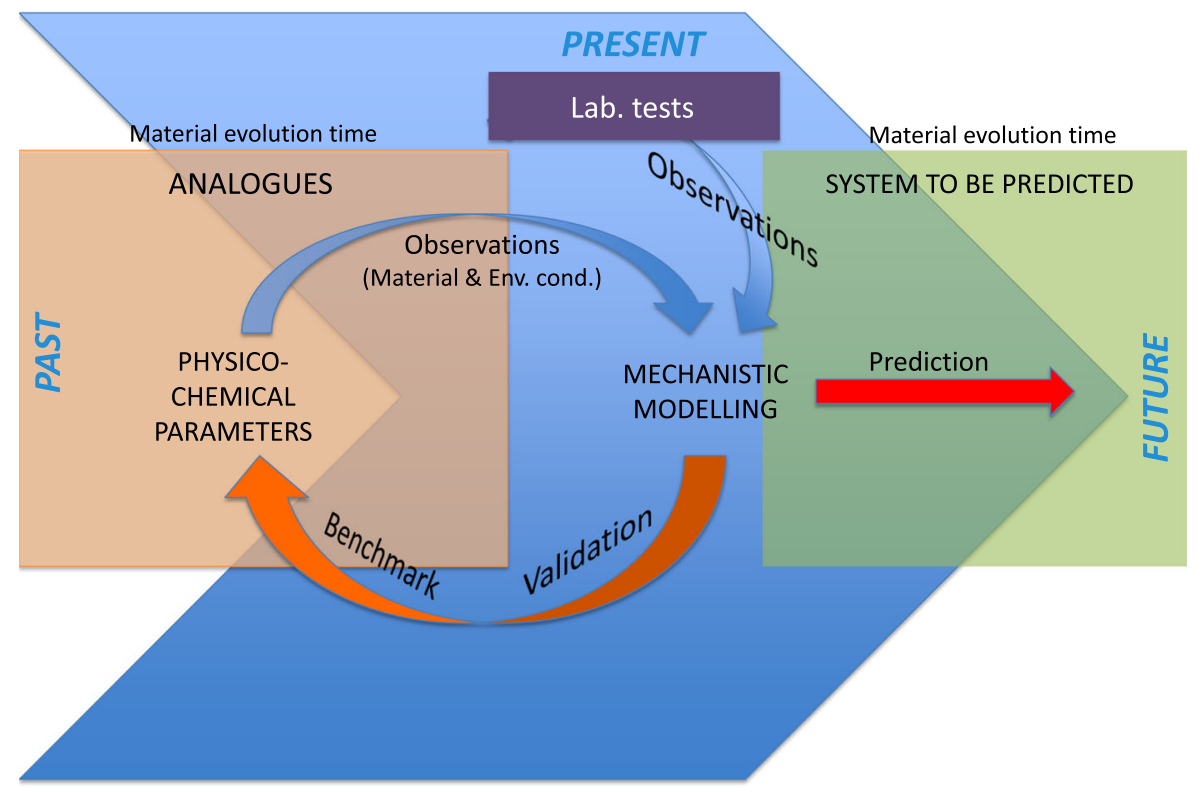

Fig. 1 Positioning of approaches using analogues in the overall prediction process

must therefore be based on two distinct objects, which share a number of characteristics: first, volcanic (or archaeological) glasses altered in a natural medium over the long term, and then nuclear glasses altered in a laboratory over a much shorter term. ${ }^{12}$ Although those materials have different compositions (aluminosilicate or soda lime glasses for natural and archaeological glasses versus borosilicate glasses for nuclear waste), past studies have shown that mechanistic and kinetic correlations are possible., The first step of the work involves studying the alteration kinetics and secondary products of natural (or archaeological) glasses experimentally over short periods to be able to correlate rate limiting mechanism with those of nuclear glasses and get the specific parameters such as activation energy, kinetic constant and its $\mathrm{pH}$ dependency for these glass compositions. ${ }^{13,14}$ Next, it must be demonstrated that kinetic models based on the experimental data extrapolated over long time scales can reflect material behaviour observed in the natural or archaeological samples. ${ }^{15}$ When a relationship can be established between the experimental data and the long-term alteration state for the natural samples, reasoning by analogy can therefore point to nuclear glass alteration models being able to predict the nuclear material behaviour over the long term. ${ }^{5}$ This is not a demonstration in the strict sense of the term, but nevertheless establishes firm links between the laboratory and the natural environment on the one hand, and between the short and long term on the other. This predictive approach would seem to present a certain degree of robustness.

For ferrous alloys (steel, cast iron, etc.) different prediction reliability durations are needed, ranging from a few decades for civil engineering structures to a thousand years for the nuclear waste package containers foreseen for repositories in several countries (France, Belgium, Japan, Switzerland,...). ${ }^{16}$ Analogues can thus be used in several ways. They may, for example, serve to validate if the corrosion kinetics deduced from short-term experiments and extrapolated to the long term are consistent with the corrosion thicknesses found on archaeological objects. Analogues can also contribute to preparing an overall curve describing the maximum corrosion rates measured in a given environment. In this case, they are the foundation for a semiempirical approach that, though limited, enables the extreme and average behaviour estimations to be narrowed. As metal corrosion processes are known to be very sensitive to environmental parameters including water oxygen content and $\mathrm{pH}$, but also the presence of bacteria, another use for analogues can be to better identify the general effects of these parameters. In a reverse approach, the nature of a metal (or alloy) structure and its influence on different local phenomena (e.g., pitting) may be examined. When the type of exposure environment of the analogue is close to that of the system seeking to be studied / predicted, the results of long-term corrosion mechanisms can be specifically targeted. This includes the presence of certain phases in the corrosion products, whether they are conductive or not, the presence of layers representing barriers for transport phenomena, and so on; allowing for direct integration into physical predictive models. Finally, and in a more exploratory way, corroded archaeological objects may contribute to understanding the non-linear behaviours of corrosion systems.

Stéphane Gin ${ }^{1}$, Philippe Dillmann ${ }^{2}$ and Nick Birbilis ${ }^{3}$ ${ }^{1}$ CEA, DEN-Marcoule, F-30207 Bagnols-sur-Cèze Cedex, France; ${ }^{2}$ LAPA-IRAMAT, NIMBE, CEA, CNRS, Université Paris-Saclay, CEA Saclay, 91191 Gif-sur-Yvette, France and ${ }^{3}$ Monash University, Department of Materials Engineering, Monash University, Clayton, VIC 3800, Australia

Correspondence: Stéphane Gin (stephane.gin@cea.fr)

\section{REFERENCES}

1. Miller, B. \& Chapman, N. Postcards from the past: archaeological and industrial analogs for deep repository materials. Radwaste Mag. 2, 32-42 (1995).

2. Miller, W. M., Chapman, N., McKinley, I., Alexander, R. \& Smellie, J. A. T. Natural Analogue Studies in the Geological Disposal of Radioactive Wastes, (Elsevier, 1994).

3. Dillmann, P., Neff, D. \& Feon, D. Archaeological analogues and corrosion prediction: from past to future. A review. Corros. Eng. Sci. Technol. 49, 567-576 (2014).

4. Sterpenich, J. \& Libourel, G. Using stained glass windows to understand the durability of toxic waste matrices. Chem. Geol. 174, 181-193 (2001).

5. Verney-Carron, A., Gin, S. \& Libourel, G. Archaeological analogs and the future of nuclear waste glass. J. Nucl. Mater. 406, 365-370 (2010).

6. Saheb, M. et al. Iron corrosion in an anoxic soil: Comparison between thermodynamic modelling and ferrous archaeological artefacts characterised along with the local in situ geochemical conditions. Appl. Geochem. 25, 1937-1948 (2010).

7. Dillmann, P. Corrosion of archaeological and heritage artefacts. Corros. Eng. Sci. Technol. 45, 313-313 (2010).

8. Dillmann, P. \& Bellot-Gurlet, L. Nanoscience and cultural heritage, (Atlantic Press, 2016). 
9. Ewing, R. C. Natural glasses: analogues for radioactive waste forms. Mat. Res. Soc. Symp. Proc. 1, 57-68 (1979).

10. Grambow, B. Nuclear waste glasses-How durable? Elements 2, 357-364 (2006).

11. Libourel, G. et al. The use of natural and archeological analogues for understanding the long-term behavior of nuclear glasses. Cr. Geosci. 343, 237-245 (2011).

12. Crovisier, J. L., Advocat, T. \& Dussossoy, J. L. Nature and role of natural alteration gels formed on the surface of ancient volcanic glasses (Natural analogs of waste containment glasses). J. Nucl. Mater. 321, 91-109 (2003).

13. Parruzot, B., Jollivet, P., Rebiscoul, D. \& Gin, S. Long-term alteration of basaltic glass: Mechanisms and rates. Geochim. Cosmochim. Acta 154, 28-48 (2015).

14. Verney-Carron, A., Gin, S. \& Libourel, G. A fractured roman glass block altered for 1800 years in seawater: analogy with nuclear waste glass in a deep geological repository. Geochim. Cosmochim. Ac 72, 5372-5385 (2008).

15. Verney-Carron, A., Gin, S., Frugier, P. \& Libourel, G. Long-term modeling of alteration-transport coupling: application to a fractured Roman glass. Geochim. Cosmochim. Acta 74, 2291-2315 (2010).
16. Cattant, F., Crusset, D. \& Feron, D. Corrosion issues in nuclear industry today. Mater. Today 11, 32-37 (2008).

(i) Open Access This article is licensed under a Creative Commons Attribution 4.0 International License, which permits use, sharing, adaptation, distribution and reproduction in any medium or format, as long as you give appropriate credit to the original author(s) and the source, provide a link to the Creative Commons license, and indicate if changes were made. The images or other third party material in this article are included in the article's Creative Commons license, unless indicated otherwise in a credit line to the material. If material is not included in the article's Creative Commons license and your intended use is not permitted by statutory regulation or exceeds the permitted use, you will need to obtain permission directly from the copyright holder. To view a copy of this license, visit http://creativecommons. org/licenses/by/4.0/.

(c) The Author(s) 2017 